\title{
Phase transition in fiber bundle models with recursive dynamics
}

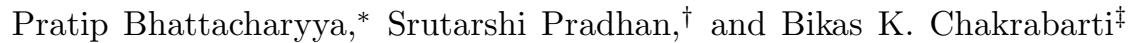 \\ Theoretical Condensed Matter Physics Division, Saha Institute of Nuclear Physics, \\ Sector - 1, Block - AF, Bidhannagar, Kolkata 700 064, India
}

(Dated: February 19, 2003)

\begin{abstract}
We study the phase transition in a class of fiber bundle models in which the fiber strengths are distributed randomly within a finite interval and global load sharing is assumed. The dynamics is expressed as recursion relations for the redistribution of the applied stress and the evolution of the surviving fraction of fibers. We show that an irreversible phase transition of second-order occurs, from a phase of partial failure to a phase of total failure, when the initial applied stress just exceeds a critical value. The phase transition is characterised by static and dynamic critical properties. We calculate exactly the critical value of the initial stress for three models of this kind, each with a different distribution of fiber strengths. We derive the exact expressions for the order parameter, the susceptibility to changes in the initial applied sress and the critical relaxation of the surviving fraction of fibers for all the three models. The static and dynamic critical exponents obtained from these expressions are found to be universal.
\end{abstract}

PACS numbers: 46.50.+a, 62.20.Mk, 64.60.Ht

\section{INTRODUCTION}

Fiber bundle models describe the collective statics and dynamics of failure in a set of fibers with random strengths under the application of a stress (force per fiber) [1, 2]. A typical model of this kind is shown schematically in Fig. [1] These models are constructed for the purpose of explaining the propagation of fractures in a loaded heterogeneous material and to determine the conditions under which it breaks completely [3, 4]. The latter requires the calculation of the strength of the bundle from the strengths of its constituent fibers which, by reasonable assumption, are drawn at random from a chosen probability distribution [1]. In some of the models [1, [5, [6] it is assumed that the load is always divided equally among all intact fibers of the bundle (global load sharing) while in other models 7, 8, 9, 10, 11] it is assumed that when a fiber breaks, the stress it was last bearing gets distributed only among the fibers next to it (local load sharing). The dynamics or propagation of fracture in a fiber bundle has been characterised in two ways: first, by the probability distribution of bursts of different sizes that occur within the bundle as the stress is gradually increased till the bundle breaks completely [6, 13, 14, 15]; second, by the of lifetime of a fiber bundle with fatigue under an applied stress 2, 11, 12]. It was suggested in 16] that the breakdown of a static fiber bundle with global load sharing can be described as a first-order phase transition, because the surviving fraction of fibers has a discontinuity at the point of breakdown. However the susceptibility to the applied stress was shown to diverge at the breakdown point and for this reason it was later suggested that the transition is of second-order [18, 19, 20, 21].

In this paper we report on the universality of the phase transition in a class of fiber bundle models. The main feature of these models is a pair of dynamical recursion relations which, similar to the formulation in [19, 20], expresses the evolution of the fiber bundle under the application of a finite stress. The strengths of the fibers are assigned randomly within a finite interval of values which is true for real fiber bundles. We study three models of this kind: in the first model the fiber strengths are distributed with uniform density; in the second model the fiber strengths are distributed with a linearly increasing density, which means that there are more strong fibers than weak ones; in the third model the fiber strengths are distributed with a linearly decreasing density so that there are more weak fibers. From the expressions of the fixed points of the dynamics we find that there is a critical initial value of the applied stress in each of the three models: on exceeding this critical value the fiber bundle undergoes an irreversible transition from a phase of partial failure to a phase of total failure. When the initial applied stress is less than or equal to the critical value, only a finite fraction of the fibers breaks as the bundle evolves to a state of mechanical equilibrium; this is the phase of partial failure. If, on the other hand, the initial applied stress is greater than the critical value, mechanical equilibrium is never reached and the entire fiber bundle eventually breaks down; this is the phase of total failure. We define an order parameter which shows that the phase transition is of second-order. As the initial applied stress approaches its

\footnotetext{
*Electronic address: pratip@cmp.saha.ernet.in

${ }^{\dagger}$ Electronic address: spradhan@cmp.saha.ernet.in

‡Electronic address: bikas@cmp.saha.ernet.in
} 
critical value from below, the order parameter is found to reduce to zero continuously following a power-law while the susceptibility of the surviving fraction of fibers to changes in the initial stress is found to diverge, also by a power-law. We derive asymptotic solutions of the dynamical recursion relations for the surviving fraction of fibers at the critical values of the initial applied stress of each model. These solutions show that the critical relaxation of the fiber bundle toward the fixed point is a power-law decay. The critical exponents in these power-laws are found to be universal, i.e., independent of the distribution of fiber strengths in the bundle.

(a)

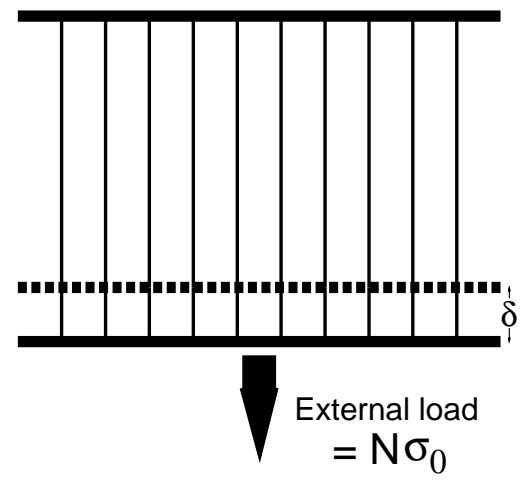

(b)

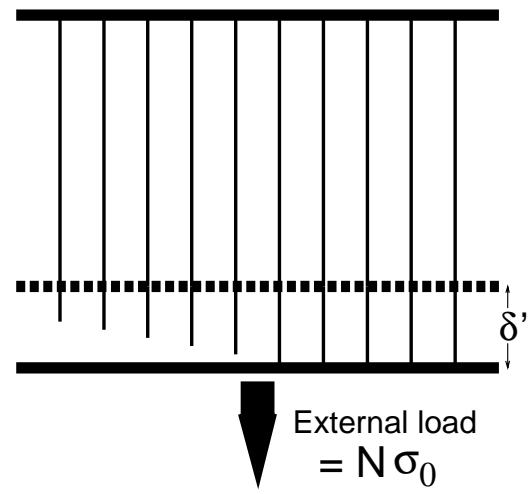

FIG. 1: Schematic diagram of a bundle of $N$ elastic fibers with random strengths, attached in parallel to a fixed plate at the top and a movable plate at the bottom. The fibers are shown as vertical solid lines and the position of the plates by bold horizontal lines. The figure shows the fibers arranged in increasing order of strength from left to right; $\sigma_{L}$ and $\sigma_{R}$ are the strengths of the weakest (extreme left) and the strongest (extreme right) fibers respectively. (a) For an initial applied stress $\sigma_{0}, \sigma_{0} \leq \sigma_{L}$, the fibers only get stretched from their relaxed position (shown by the bold broken line); the strain $\delta$ measures the elastic deformation of the bundle. (b) For $\sigma_{L}<\sigma_{0} \leq \sigma_{R}$, the fibers begin to break causing a plastic deformation of the bundle (now given by the strain $\delta^{\prime}$ ); the figure schematically shows the strained positions of the broken fibers at the time of their breaking in order to give an impression of successive failures. When $\sigma_{0}>\sigma_{R}$, all fibers in the bundle break at once.

\section{DYNAMICS OF THE FIBER BUNDLE}

We consider fiber bundle models with global load sharing approximation, i.e., any force applied on a bundle is shared equally by all the intact fibers in it. The strength of each fiber is determined by a threshold value $\sigma_{\text {thresh }}$ of the stress that it can bear, beyond which the fiber breaks. The threshold stress of the fibers in the bundle are distributed randomly with a normalised density $\rho\left(\sigma_{\text {thresh }}\right)$ within a finite interval $\left[\sigma_{L}, \sigma_{R}\right]$ where $\sigma_{L}$ and $\sigma_{R}$ are respectively the strengths of the weakest and the strongest fiber in the bundle:

$$
\int_{\sigma_{L}}^{\sigma_{R}} \rho\left(\sigma_{\text {thresh }}\right) \mathrm{d} \sigma_{\text {thresh }}=1 .
$$


The probability distribution of the threshold stress is given by:

$$
P\left(\sigma_{\text {thresh }}\right)= \begin{cases}0, & 0 \leq \sigma_{\text {thresh }}<\sigma_{L} \\ \int_{\sigma_{L}}^{\sigma_{\text {thresh }}} \rho(\sigma) \mathrm{d} \sigma, & \sigma_{L} \leq \sigma_{\text {thresh }} \leq \sigma_{R} \\ 1, & \sigma_{R}<\sigma_{\text {thresh }} .\end{cases}
$$

We study the breaking dynamics of the model under the application of an initial stress $\sigma_{0}$ (for example, by attaching an external load to the bottom plate in Fig. 1) where the stress is defined as the force exerted per fiber. The fibers whose strengths are less than $\sigma_{0}$ break immediately. Following this initial rupture the applied stress gets redistributed among the surviving fibers. Since the number of fibers supporting the load has decreased the redistributed stress is greater than the initial stress and this causes further breaking of fibers. The process continues till a state of mechanical equilibrium is reached, where the surviving fibers have strengths greater than the redistributed stress, or till all fibers in the bundle are broken. The state of mechanical equilibrium, if it exists, appears as a fixed point of the model under the assigned dynamics.

The nature of the breaking dynamics allows it to be represented as a recursion relation operating in discrete timesteps [17, 19, 20]. If $U_{t}$ is the fraction of fibers in the initial bundle that survive after time-step $t$, the redistributed stress due to global load sharing after $t$ time-steps is

$$
\sigma_{t}=\frac{\sigma_{0}}{U_{t}} .
$$

After $t+1$ time-steps the surviving fraction of fibers becomes

$$
U_{t+1}=1-P\left(\sigma_{t}\right)
$$

In a real bundle comprising of a finite number of fibers there will be fluctuations in the local density of fiber strengths as well as the load sharing. Such fluctuatins are ignored in the contruction of Eq. (3) and Eq. (4). It follows from Eq. (3) and Eq. (4) that the quantities $\sigma_{t}$ and $U_{t}$ evolve by the recurrences

$$
\sigma_{t+1}=\frac{\sigma_{0}}{1-P\left(\sigma_{t}\right)}
$$

and

$$
U_{t+1}=1-P\left(\sigma_{0} / U_{t}\right), \quad U_{0}=1,
$$

which formally define the dynamics of this class of models. With the probability distribution Eq. (2), the recursion relation (66) clearly shows that none of the fibers break for an initial stress $\sigma_{0} \leq \sigma_{L}$, while on application of $\sigma_{0}>\sigma_{R}$, all fibers in the bundle break simultaneously.

The fixed points [22] of the model, $\sigma^{*}$ and $U^{*}$, are determined by the relations:

$$
\sigma^{*}\left[1-P\left(\sigma^{*}\right)\right]=\sigma_{0}
$$

and

$$
U^{*}+P\left(\sigma_{0} / U^{*}\right)=1
$$

Though the quantities $\sigma_{t}$ and $U_{t}$ evolve in time till they reach their fixed point values, Eq. (3) shows that their product $\sigma_{t} U_{t}$ is a constant of motion, always equal to the initial value $\sigma_{0}$. All static and dynamic properties of the models are consequences of this invariance.

\section{CRITICAL PROPERTIES FOR UNIFORM DENSITY OF FIBER STRENGTHS}

We consider first the case where the random strengths $\sigma_{\text {thresh }}$ of the fibers are distributed with uniform density in the interval $\left[\sigma_{L}, \sigma_{R}\right]$. The normalised density function is: 


$$
\rho\left(\sigma_{\text {thresh }}\right)= \begin{cases}0, & 0 \leq \sigma_{\text {thresh }}<\sigma_{L} \\ \frac{1}{\sigma_{R}-\sigma_{L}}, & \sigma_{L} \leq \sigma_{\text {thresh }} \leq \sigma_{R} \\ 0, & \sigma_{R}<\sigma_{\text {thresh }}\end{cases}
$$

and the probability distribution, by the definition in Eq. (2), is given by (Fig. 2):

$$
P\left(\sigma_{\text {thresh }}\right)= \begin{cases}0, & 0 \leq \sigma_{\text {thresh }}<\sigma_{L} \\ \frac{\sigma_{\text {thresh }}-\sigma_{L}}{\sigma_{R}-\sigma_{L}}, & \sigma_{L} \leq \sigma_{\text {thresh }} \leq \sigma_{R} \\ 1, & \sigma_{R}<\sigma_{\text {thresh }} .\end{cases}
$$

We consider only $\sigma_{R}>\sigma_{L}$ as the case of $\sigma_{R}=\sigma_{L}$ is trivial.

(a)

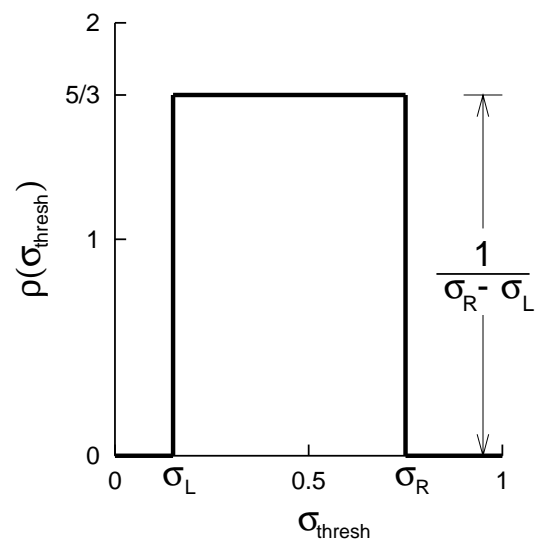

(b)

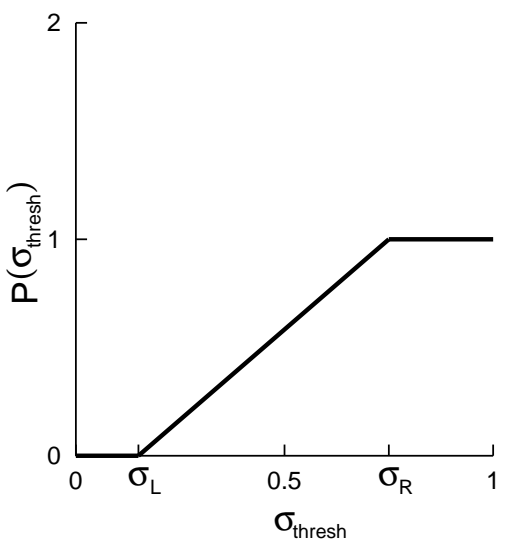

FIG. 2: (a) The density function $\rho$ and (b) the probability distribution $P$ of random fiber strengths $\sigma_{\text {thresh }}$ distributed with uniform density in the interval $\left[\sigma_{L}, \sigma_{R}\right]$. In the particular instance shown in the figure $\sigma_{L}=0.15$ and $\sigma_{R}=0.75$.

With this particular choice of the distribution of fiber strengths the recursion relations (50) and (6), for an initial stress $\sigma_{L} \leq \sigma_{0} \leq \sigma_{R}$, appear as:

$$
\sigma_{t+1}=\sigma_{0}\left(\frac{\sigma_{R}-\sigma_{L}}{\sigma_{R}-\sigma_{t}}\right)
$$

and

$$
U_{t+1}=\frac{1}{\sigma_{R}-\sigma_{L}}\left(\sigma_{R}-\frac{\sigma_{0}}{U_{t}}\right), \quad U_{0}=1 .
$$

The fixed point equations, obtained from Eq. (7) and Eq. (8), appear in quadratic form:

$$
\left(\sigma^{*}\right)^{2}-\sigma_{R} \sigma^{*}+\sigma_{0}\left(\sigma_{R}-\sigma_{L}\right)=0
$$

and

$$
\left(U^{*}\right)^{2}-\left(\frac{\sigma_{R}}{\sigma_{R}-\sigma_{L}}\right) U^{*}+\frac{\sigma_{0}}{\sigma_{R}-\sigma_{L}}=0
$$


Consequently each of the recurrences, (11) and (12), have two fixed points:

$$
\begin{gathered}
\sigma_{1,2}^{*}=\frac{\sigma_{R}}{2} \pm\left(\sigma_{R}-\sigma_{L}\right)^{1 / 2}\left[\frac{\sigma_{R}^{2}}{4\left(\sigma_{R}-\sigma_{L}\right)}-\sigma_{0}\right]^{1 / 2}, \\
U_{1,2}^{*}=\frac{\sigma_{R}}{2\left(\sigma_{R}-\sigma_{L}\right)} \pm \frac{1}{\left(\sigma_{R}-\sigma_{L}\right)^{1 / 2}}\left[\frac{\sigma_{R}^{2}}{4\left(\sigma_{R}-\sigma_{L}\right)}-\sigma_{0}\right]^{1 / 2} .
\end{gathered}
$$

The subscripts 1 and 2 stand for the expressions containing the plus and the minus sign respectively. While $\sigma_{2}^{*}$ and $U_{1}^{*}$ are stable fixed points, $\sigma_{1}^{*}$ and $U_{2}^{*}$ are unstable (Fig. 33). It is clear that the fixed points for the redistributed stress and the surviving fraction of fibers are related by:

$$
U_{1,2}^{*}=\frac{\sigma_{R}-\sigma_{2,1}^{*}}{\sigma_{R}-\sigma_{L}}
$$

(a)

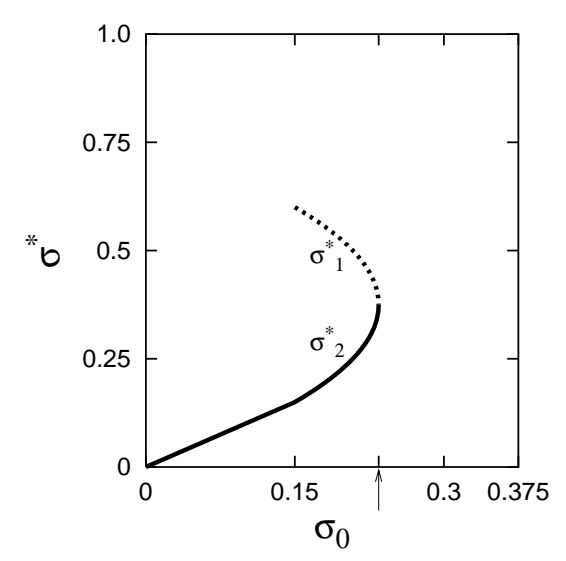

(b)

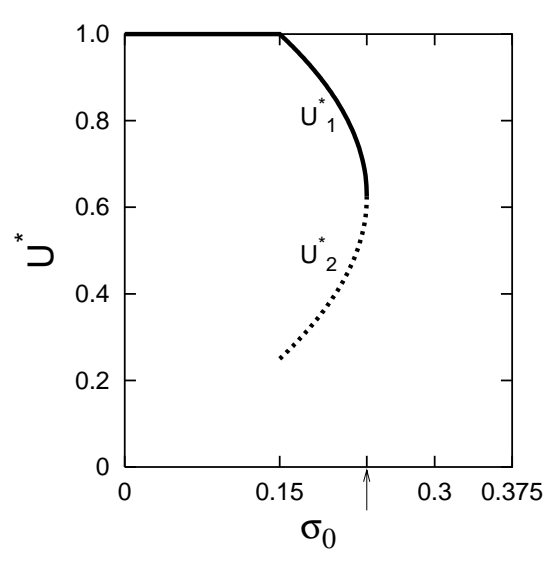

FIG. 3: The fixed points of (a) the redistributed stress, and (b) the surviving fraction of fibers for the particular probability distribution of fiber strengths shown in Fig. 2 In each part of the figure the curve for the stable fixed points is shown by a bold solid line and that for the unstable fixed points are shown by a bold broken line. As in Fig. 2 we have $\sigma_{L}=0.15$ and $\sigma_{R}=0.75$, so that $\sigma_{0}^{\text {crit }}=0.234375$; the position of the critical point is marked by an arrowhead. For $\sigma_{0} \leq \sigma_{L}$ the fixed points are trivial: since there are no broken fibers $\sigma^{*}=\sigma_{0}$ and $U^{*}=U_{0}=1$.

The quadratic equations (13) and (14) show that the initial applied stress $\sigma_{0}$ has a critical value:

$$
\sigma_{0}^{\text {crit }}=\frac{\sigma_{R}^{2}}{4\left(\sigma_{R}-\sigma_{L}\right)}
$$

at which their discriminants become zero and only a stable fixed point exists. Since both $\sigma$ and $U$ have physical meanings, a state of mechanical equilibrium exists if the quantities $\sigma_{2}^{*}$ and $U_{1}^{*}$ are positive real-valued; this happens only when $\sigma_{0} \leq \sigma_{0}^{\text {crit }}$. For $\sigma_{0}>\sigma_{0}^{\text {crit }}$, there are no real-valued fixed points - the dynamics will continue till all fibers in the bundle are broken. Therefore a transition ocurs just above $\sigma_{0}^{\text {crit }}$ from a phase of partial failure of the bundle in equilibrium to a phase of total failure. The order parameter $\mathcal{O}$ for this phase transition is defined in terms of the stable fixed point for the surviving fraction of fibers: 


$$
\mathcal{O} \equiv U_{1}^{*}-U_{1-\text { crit }}^{*}, \quad \sigma_{L} \leq \sigma_{0} \leq \sigma_{0}^{\text {crit }}
$$

where

$$
U_{1-\text { crit }}^{*}=\frac{\sigma_{R}}{2\left(\sigma_{R}-\sigma_{L}\right)}
$$

is the value of $U_{1}^{*}$ under the critical initial stress. As Eq. (16) shows, the order parameter goes to zero continuously by a power law as $\sigma_{0}$ approaches its critical value from below:

$$
\mathcal{O}=\left(\frac{\sigma_{0}^{\text {crit }}-\sigma_{0}}{\sigma_{R}-\sigma_{L}}\right)^{1 / 2}, \quad \sigma_{L} \leq \sigma_{0} \leq \sigma_{0}^{\text {crit }}
$$

It is obvious that the critical value must have the lower bound:

$$
\sigma_{0}^{\text {crit }} \geq \sigma_{L}
$$

which, for the expression given in Eq. (18), requires that

$$
\sigma_{R} \geq 2 \sigma_{L}
$$

The above condition (23) in turn imposes an upper bound on the critical value of the initial stress:

$$
\sigma_{0}^{\text {crit }} \leq \frac{\sigma_{R}}{2} .
$$

Static critical behaviour of the fiber bundle is observed in the susceptibility of the fixed point of the surviving fraction of fibers to changes in the initial stress $\sigma_{0}$. From Eq. (16) we see that the susceptibility diverges in the form of a power law as the initial applied stress approaches its critical value from below:

$$
\chi=\left|\frac{\mathrm{d} U_{1}^{*}}{\mathrm{~d} \sigma_{0}}\right| \propto\left(\sigma_{0}^{\text {crit }}-\sigma_{0}\right)^{-1 / 2}, \quad \sigma_{L} \leq \sigma_{0} \leq \sigma_{0}^{\text {crit }} .
$$

Dynamical critical behaviour is observed in the process of relaxation of the fiber bundle to a fixed point. At the critical point $\sigma_{0}=\sigma_{0}^{\text {crit }}$ the evolution of the surviving fraction of fibers by (12) is reduced to the following recurrence:

$$
U_{t+1}=\frac{\sigma_{R}}{\sigma_{R}-\sigma_{L}}\left[1-\frac{\sigma_{R}}{4\left(\sigma_{R}-\sigma_{L}\right)} \frac{1}{U_{t}}\right], \quad U_{0}=1,
$$

and its only fixed point, a stable one, is given by Eq. (20).

The recurrence (26) has a closed-form solution:

$$
U_{t}-U_{1-\text { crit }}^{*}=\frac{U_{1-\text { crit }}^{*}\left(1-U_{1-\text { crit }}^{*}\right)}{1+\left(1-U_{1-\text { crit }}^{*}\right)(t-1)}
$$

Thus the asymptotic behaviour of the surviving fraction of fibers is a power law decay - a critically slow relaxation to the fixed point:

$$
\begin{aligned}
U_{t}-U_{1-\text { crit }}^{*} & \sim \frac{U_{1-\text { crit }}^{*}}{t}, \quad t \rightarrow \infty \\
& \sim \frac{1}{2}\left(\frac{\sigma_{R}}{\sigma_{R}-\sigma_{L}}\right) \frac{1}{t} .
\end{aligned}
$$

A special case of this model, with $\sigma_{L}=0$ and $\sigma_{R}=1$, was studied in [23]. The critical properties obtained in [23] can now be derived easily from the general results of this section. 


\section{CRITICAL PROPERTIES FOR LINEARLY INCREASING DENSITY OF FIBER STRENGTHS}

We consider next the case where the random strengths $\sigma_{\text {thresh }}$ of the fibers are distributed with linearly increasing density in the interval $\left[\sigma_{L}, \sigma_{R}\right], \sigma_{R}>\sigma_{L}$. The normalised density function and the probability distribution of the fiber strengths are given by (illustrated in Fig. 4):

$$
\rho\left(\sigma_{\text {thresh }}\right)= \begin{cases}0, & 0 \leq \sigma_{\text {thresh }}<\sigma_{L} \\ \frac{2\left(\sigma_{\text {thresh }}-\sigma_{L}\right)}{\left(\sigma_{R}-\sigma_{L}\right)^{2}}, & \sigma_{L} \leq \sigma_{\text {thresh }} \leq \sigma_{R} \\ 0, & \sigma_{R}<\sigma_{\text {thresh }}\end{cases}
$$

and

$$
P\left(\sigma_{\text {thresh }}\right)= \begin{cases}0, & 0 \leq \sigma_{\text {thresh }}<\sigma_{L} \\ \left(\frac{\sigma_{\text {thresh }}-\sigma_{L}}{\sigma_{R}-\sigma_{L}}\right)^{2}, & \sigma_{L} \leq \sigma_{\text {thresh }} \leq \sigma_{R} \\ 1, & \sigma_{R}<\sigma_{\text {thresh }}\end{cases}
$$

(a)

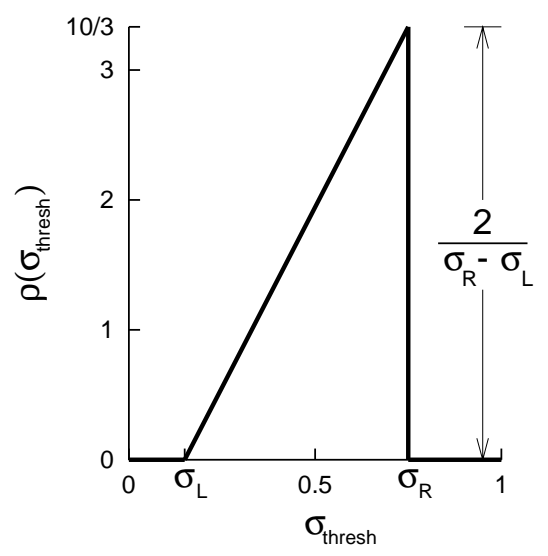

(b)

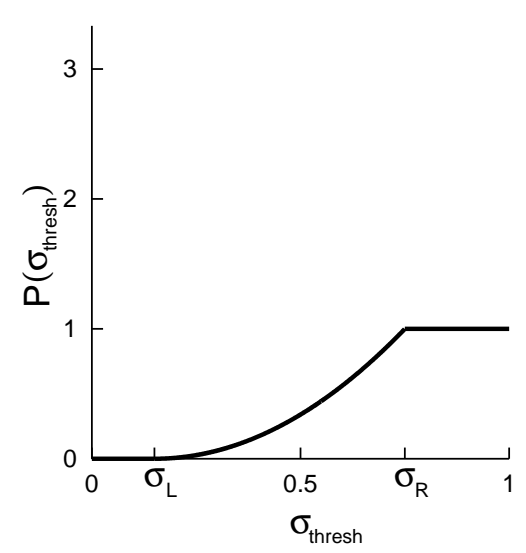

FIG. 4: (a) The density function $\rho$ and (b) the probability distribution $P$ of random fiber strengths $\sigma_{\text {thresh }}$ distributed with linearly increasing density in the interval $\left[\sigma_{L}, \sigma_{R}\right]$. In the particular instance shown in the figure $\sigma_{L}=0.15$ and $\sigma_{R}=0.75$.

Here we introduce the transformed quantities:

$$
\Gamma_{0}=\frac{\sigma_{0}}{\sigma_{R}-\sigma_{L}}, \quad \Gamma_{L}=\frac{\sigma_{L}}{\sigma_{R}-\sigma_{L}}, \quad \Gamma_{t}=\frac{\sigma_{t}}{\sigma_{R}-\sigma_{L}}
$$

For an initial stress $\sigma_{L} \leq \sigma_{0} \leq \sigma_{R}$ (or, $\Gamma_{L} \leq \Gamma_{0} \leq \Gamma_{L}+1$ ) along with the distribution of fiber strengths given by Eq. (30), the recursion relations (5) and (6) appear as:

$$
\Gamma_{t+1}=\frac{\Gamma_{0}}{1-\left(\Gamma_{t}-\Gamma_{L}\right)^{2}}
$$

and

$$
U_{t+1}=1-\left(\frac{\Gamma_{0}}{U_{t}}-\Gamma_{L}\right)^{2}, \quad U_{0}=1
$$


The fixed point equations, (7) and (8), now assume cubic form:

$$
\left(\Gamma^{*}\right)^{3}-2 \Gamma_{L}\left(\Gamma^{*}\right)^{2}+\left(\Gamma_{L}^{2}-1\right) \Gamma^{*}+\Gamma_{0}=0
$$

where $\Gamma^{*}=\sigma^{*} /\left(\sigma_{R}-\sigma_{L}\right)$, and

$$
\left(U^{*}\right)^{3}+\left(\Gamma_{L}^{2}-1\right)\left(U^{*}\right)^{2}-\left(2 \Gamma_{L} \Gamma_{0}\right) U^{*}+\Gamma_{0}^{2}=0 .
$$

Consequently each of the recurrences (32) and (33) have three fixed points - only one in each case is found to be stable. For the redistributed stress the fixed points are:

$$
\begin{aligned}
\Gamma_{1}^{*} & =\frac{2}{3} \Gamma_{L}+2 K \cos \frac{\Phi}{3}, \\
\Gamma_{2}^{*} & =\frac{2}{3} \Gamma_{L}-K \cos \frac{\Phi}{3}+\sqrt{3} K \sin \frac{\Phi}{3}, \\
\Gamma_{3}^{*} & =\frac{2}{3} \Gamma_{L}-K \cos \frac{\Phi}{3}-\sqrt{3} K \sin \frac{\Phi}{3},
\end{aligned}
$$

where

$$
K=\frac{1}{3} \sqrt{3+\Gamma_{L}^{2}}
$$

and

$$
\cos \Phi=\frac{\Gamma_{L}\left(9-\Gamma_{L}^{2}\right)-27 \Gamma_{0} / 2}{\left(3+\Gamma_{L}^{2}\right)^{3 / 2}} .
$$

Similarly, for the surviving fraction of fibers the fixed points are:

$$
\begin{aligned}
U_{1}^{*} & =\frac{1-\Gamma_{L}^{2}}{3}+2 J \cos \frac{\Theta}{3}, \\
U_{2}^{*} & =\frac{1-\Gamma_{L}^{2}}{3}-J \cos \frac{\Theta}{3}+\sqrt{3} J \sin \frac{\Theta}{3}, \\
U_{3}^{*} & =\frac{1-\Gamma_{L}^{2}}{3}-J \cos \frac{\Theta}{3}-\sqrt{3} J \sin \frac{\Theta}{3},
\end{aligned}
$$

where

$$
J=\frac{1}{3} \sqrt{\left(\Gamma_{L}^{2}-1\right)^{2}+6 \Gamma_{L} \Gamma_{0}}
$$

and

$$
\cos \Theta=\frac{\left(1-\Gamma_{L}^{2}\right)\left[\left(\Gamma_{L}^{2}-1\right)^{2}+9 \Gamma_{L} \Gamma_{0}\right]-27 \Gamma_{0}^{2} / 2}{\left[\left(\Gamma_{L}^{2}-1\right)^{2}+6 \Gamma_{L} \Gamma_{0}\right]^{3 / 2}} .
$$

Of these fixed points $\Gamma_{2}^{*}$ and $U_{1}^{*}$ are stable whereas $\Gamma_{1}^{*}, \Gamma_{3}^{*}$ and $U_{2}^{*}, U_{3}^{*}$ are unstable (Fig. 囵).

Similar to the case in the previous section the discriminants of the cubic equations (34) and (35) become zero at a critical value $\sigma_{0}^{\text {crit }}$ (or, $\Gamma_{0}^{\text {crit }}$ ) of the initial applied stress:

$$
\begin{aligned}
\Gamma_{0}^{\text {crit }} & =\frac{\sigma_{0}^{\text {crit }}}{\sigma_{R}-\sigma_{L}} \\
& =\frac{2}{27}\left[\Gamma_{L}\left(9-\Gamma_{L}^{2}\right)+\left(3+\Gamma_{L}^{2}\right)^{3 / 2}\right]
\end{aligned}
$$


(a)

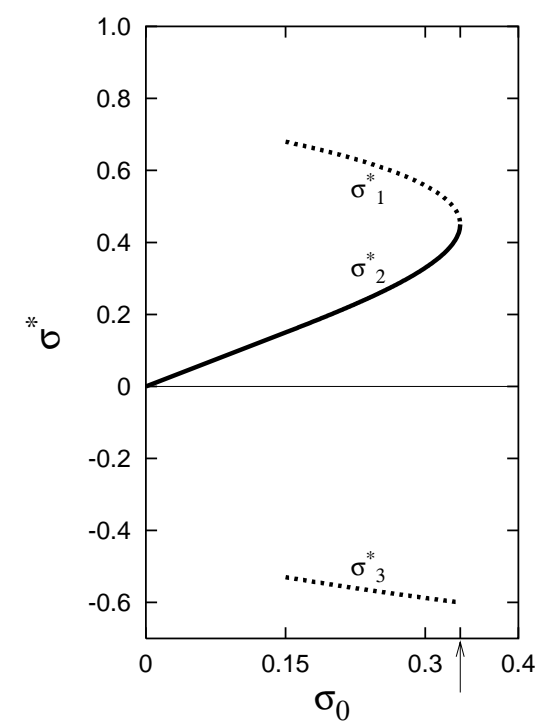

(b)

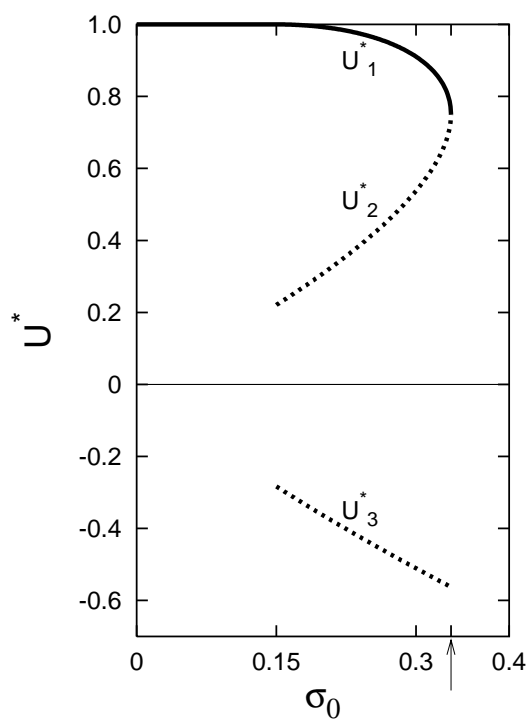

FIG. 5: The fixed points of (a) the redistributed stress, and (b) the surviving fraction of fibers for the probability distribution of fiber strengths shown in Fig. 4 In each part of the figure the curve for the stable fixed points is shown by a bold solid line and those for the unstable fixed points are shown by bold broken lines. We have $\sigma_{L}=0.15$ and $\sigma_{R}=0.75$, so that $\sigma_{0}^{\text {crit }}=0.3375$; the position of the critical point is marked by an arrowhead. As in the previous case, for $\sigma_{0} \leq \sigma_{L}$ the fixed points are trivial: since there are no broken fibers $\sigma^{*}=\sigma_{0}$ and $U^{*}=U_{0}=1$.

and then each of the quantities $\Gamma$ and $U$ have one stable and one unstable fixed point. As before the critical point has the trivial lower bound:

$$
\sigma_{0}^{\text {crit }} \geq \sigma_{L}
$$

The expression of $\Gamma_{0}^{\text {crit }}$ in Eq. (46) shows that it approaches the lower bound as $\Gamma_{L} \rightarrow \infty$ which happens for finite values of $\sigma_{L}$ and $\sigma_{R}$ when $\left(\sigma_{R}-\sigma_{L}\right) \rightarrow 0$. It follows that the upper bound for the critical point is also trivial:

$$
\sigma_{0}^{\text {crit }} \leq \sigma_{R}
$$

which is different from the condition (24) for the case of uniform distribution. At the critical point we get from Eq. (40) and Eq. (45):

$$
\cos \Phi_{\text {crit }}=\cos \Theta_{\text {crit }}=-1
$$

or,

$$
\Phi_{\text {crit }}=\Theta_{\text {crit }}=\pi
$$

The stable fixed points $\Gamma_{2}^{*}$ and $U_{1}^{*}$ are positive real-valued when $\Gamma_{0} \leq \Gamma_{0}^{\text {crit }}$; thus the fiber bundle always reaches a state of mechanical equilibrium after partial failure under an initial applied stress $\sigma_{0} \leq \sigma_{0}^{\text {crit }}$. For $\sigma_{0}>\sigma_{0}^{\text {crit }}$ (or, $\left.\Gamma_{0}>\Gamma_{0}^{\text {crit }}\right), \Gamma_{2}^{*}$ and $U_{1}^{*}$ are no longer real-valued and the entire fiber bundle eventually breaks down. The transition from the phase of partial failure to the phase of total failure takes place when $\sigma_{0}$ just exceeds $\sigma_{0}^{\text {crit }}$ and the order parameter for this phase transition is defined as in Eq. (19):

$$
\mathcal{O} \equiv U_{1}^{*}-U_{1-\text { crit }}^{*}
$$


Close to the critical point but below it, we can write, from Eq. (45) and Eq. (50), that:

$$
\begin{aligned}
\pi-\Theta & \simeq \sin \Theta \\
& \simeq \frac{3 \sqrt{3} \Gamma_{0}^{\text {crit }}\left(3+\Gamma_{L}^{2}\right)^{3 / 4}\left(\Gamma_{0}^{\text {crit }}-\Gamma_{0}\right)^{1 / 2}}{\left[\left(\Gamma_{L}^{2}-1\right)^{2}+6 \Gamma_{L} \Gamma_{0}^{\text {crit }}\right]^{3 / 2}}
\end{aligned}
$$

and the expressions for the fixed points in Eq. (41) and Eq. (42) reduce to the forms:

$$
U_{1}^{*} \simeq U_{1-\text { crit }}^{*}+\frac{\Gamma_{0}^{\text {crit }}\left(3+\Gamma_{L}^{2}\right)^{3 / 4}}{\left(\Gamma_{L}^{2}-1\right)^{2}+6 \Gamma_{L} \Gamma_{0}^{\text {crit }}}\left(\Gamma_{0}^{\text {crit }}-\Gamma_{0}\right)^{1 / 2}
$$

and

$$
U_{2}^{*} \simeq U_{2-\text { crit }}^{*}-\frac{\Gamma_{0}^{\mathrm{crit}}\left(3+\Gamma_{L}^{2}\right)^{3 / 4}}{\left(\Gamma_{L}^{2}-1\right)^{2}+6 \Gamma_{L} \Gamma_{0}^{\text {crit }}}\left(\Gamma_{0}^{\text {crit }}-\Gamma_{0}\right)^{1 / 2}
$$

where

$$
U_{1-\mathrm{crit}}^{*}=U_{2-\mathrm{crit}}^{*}=\frac{1-\Gamma_{L}^{2}}{3}+\frac{1}{3} \sqrt{\left(\Gamma_{L}^{2}-1\right)^{2}+6 \Gamma_{L} \Gamma_{0}^{\mathrm{crit}}}
$$

is the stable fixed point value of the surviving fraction of fibers under the critical initial stress $\sigma_{0}^{\text {crit }}$. Therefore, following the definition of the order parameter in Eq. (51) we get from the above equation:

$$
\mathcal{O}=\frac{\Gamma_{0}^{\mathrm{crit}}\left(3+\Gamma_{L}^{2}\right)^{3 / 4}}{\left(\Gamma_{L}^{2}-1\right)^{2}+6 \Gamma_{L} \Gamma_{0}^{\mathrm{crit}}}\left(\Gamma_{0}^{\mathrm{crit}}-\Gamma_{0}\right)^{1 / 2}, \quad \Gamma_{0} \rightarrow \Gamma_{0}^{\mathrm{crit}}-.
$$

On replacing the transformed variable $\Gamma_{0}$ by the original $\sigma_{0}$, Eq. (56) shows that the order parameter goes to zero continuously following the same power-law as in Eq. (21) for the previous case when $\sigma_{0}$ approaches its critical value from below.

Similarly the susceptibility diverges by the same power-law as in Eq. (25) on approaching the critical point from below:

$$
\chi=\left|\frac{\mathrm{d} U_{1}^{*}}{\mathrm{~d} \sigma_{0}}\right| \propto\left(\Gamma_{0}^{\mathrm{crit}}-\Gamma_{0}\right)^{-1 / 2}, \quad \Gamma_{0} \rightarrow \Gamma_{0}^{\mathrm{crit}}-.
$$

The critical dynamics of the fiber bundle is given by the asymptotic closed form solution of the recurrence (33) for $\Gamma_{0}=\Gamma_{0}^{\text {crit }}$ :

$$
U_{t}-U_{1-\text { crit }}^{*} \sim\left[\frac{\left(U_{1-\text { crit }}^{*}\right)^{4}}{3\left(\Gamma_{0}^{\text {crit }}\right)^{2}-2 \Gamma_{L} \Gamma_{0}^{\text {crit }} U_{1-\text { crit }}^{*}}\right] \frac{1}{t}, \quad t \rightarrow \infty,
$$

where $\Gamma_{0}^{\text {crit }}$ and $U_{1-\text { crit }}^{*}$ are given in Eq. (46) and Eq. (55) respectively. This shows that the asymptotic relaxation of the surviving fraction of fibers to its stable fixed point under the critical initial stress has the same inverse of time form as found in the case of uniform density of fiber strengths [Eq. (28)].

\section{CRITICAL PROPERTIES FOR LINEARLY DECREASING DENSITY OF FIBER STRENGTHS}

Contrary to the case of the previoius section we now consider a fiber bundle where the random threshold values are distributed with a linearly decreasing density in the interval $\left[\sigma_{L}, \sigma_{R}\right], \sigma_{R}>\sigma_{L}$. Instead of Eq. (29) and Eq. (30) we now have the following normalised density function and probability distribution (illustrated in Fig. (6):

$$
\rho\left(\sigma_{\text {thresh }}\right)=\left\{\begin{array}{lc}
0, & 0 \leq \sigma_{\text {thresh }}<\sigma_{L} \\
\frac{2\left(\sigma_{R}-\sigma_{\text {thresh }}\right)}{\left(\sigma_{R}-\sigma_{L}\right)^{2}}, & \sigma_{L} \leq \sigma_{\text {thresh }} \leq \sigma_{R} \\
0, & \sigma_{R}<\sigma_{\text {thresh }}
\end{array}\right.
$$


and

$$
P\left(\sigma_{\text {thresh }}\right)=\left\{\begin{array}{lc}
0, & 0 \leq \sigma_{\text {thresh }}<\sigma_{L} \\
1-\left(\frac{\sigma_{R}-\sigma_{\text {thresh }}}{\sigma_{R}-\sigma_{L}}\right)^{2}, & \sigma_{L} \leq \sigma_{\text {thresh }} \leq \sigma_{R} \\
1, & \sigma_{R}<\sigma_{\text {thresh }}
\end{array}\right.
$$

(a)

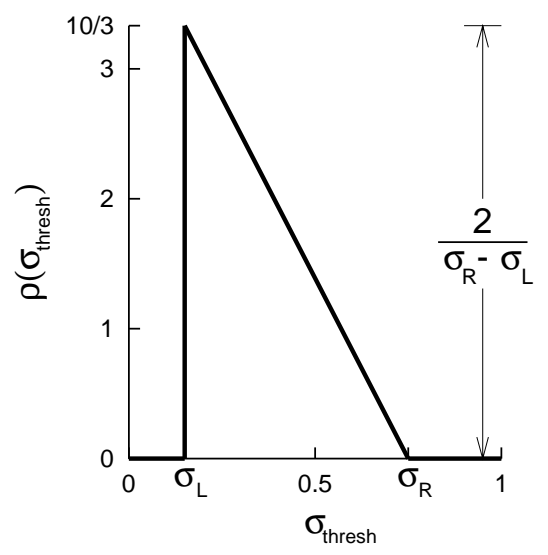

(b)

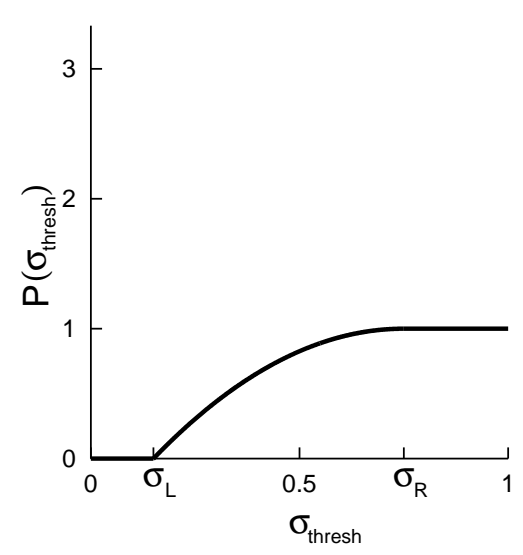

FIG. 6: (a) The density function $\rho$ and (b) the probability distribution $P$ of random fiber strengths $\sigma_{\text {thresh }}$ distributed with linearly decreasing density in the interval $\left[\sigma_{L}, \sigma_{R}\right]$. Similar to the cases shown in Fig. 2 and Fig. 4 we have $\sigma_{L}=0.15$ and $\sigma_{R}=0.75$ in this example also.

With the transformed quantities defined in Eq. (31) the recurrences (15) and (6) for $\sigma_{L} \leq \sigma_{0} \leq \sigma_{R}$ appear as:

$$
\Gamma_{t+1}=\frac{\Gamma_{0}}{\left(1+\Gamma_{L}-\Gamma_{t}\right)^{2}}
$$

and

$$
U_{t+1}=\left(1+\Gamma_{L}-\frac{\Gamma_{0}}{U_{t}}\right)^{2}, \quad U_{0}=1 .
$$

The fixed point equations are again cubic:

$$
\begin{gathered}
\left(\Gamma^{*}\right)^{3}-2\left(1+\Gamma_{L}\right)\left(\Gamma^{*}\right)^{2}+\left(1+\Gamma_{L}\right)^{2} \Gamma^{*}-\Gamma_{0}=0, \\
\left(U^{*}\right)^{3}-\left(1+\Gamma_{L}\right)^{2}\left(U^{*}\right)^{2}+2\left(1+\Gamma_{L}\right) \Gamma_{0} U^{*}-\Gamma_{0}^{2}=0
\end{gathered}
$$

and they have the following solutions:

$$
\begin{aligned}
\Gamma_{1}^{*} & =\frac{2}{3}\left(1+\Gamma_{L}\right)+2 K^{\prime} \cos \frac{\Phi^{\prime}}{3}, \\
\Gamma_{2}^{*} & =\frac{2}{3}\left(1+\Gamma_{L}\right)-K^{\prime} \cos \frac{\Phi^{\prime}}{3}+\sqrt{3} K^{\prime} \sin \frac{\Phi^{\prime}}{3}, \\
\Gamma_{3}^{*} & =\frac{2}{3}\left(1+\Gamma_{L}\right)-K^{\prime} \cos \frac{\Phi^{\prime}}{3}-\sqrt{3} K^{\prime} \sin \frac{\Phi^{\prime}}{3},
\end{aligned}
$$


where

$$
\begin{gathered}
K^{\prime}=\frac{1+\Gamma_{L}}{3}, \\
\cos \Phi^{\prime}=\frac{27 \Gamma_{0}}{2\left(1+\Gamma_{L}\right)^{3}}-1
\end{gathered}
$$

and

$$
\begin{aligned}
& U_{1}^{*}=\frac{\left(1+\Gamma_{L}\right)^{2}}{3}+2 J^{\prime} \cos \frac{\Theta^{\prime}}{3} \\
& U_{2}^{*}=\frac{\left(1+\Gamma_{L}\right)^{2}}{3}-J^{\prime} \cos \frac{\Theta^{\prime}}{3}+\sqrt{3} J^{\prime} \sin \frac{\Theta^{\prime}}{3} \\
& U_{3}^{*}=\frac{\left(1+\Gamma_{L}\right)^{2}}{3}-J^{\prime} \cos \frac{\Theta^{\prime}}{3}-\sqrt{3} J^{\prime} \sin \frac{\Theta^{\prime}}{3},
\end{aligned}
$$

where

$$
\begin{gathered}
J^{\prime}=\frac{1}{3} \sqrt{\left(1+\Gamma_{L}\right)^{4}-6\left(1+\Gamma_{L}\right) \Gamma_{0}}, \\
\cos \Theta^{\prime}=\frac{\left(1+\Gamma_{L}\right)^{3}\left[\left(1+\Gamma_{L}\right)^{3}-9 \Gamma_{0}\right]+27 \Gamma_{0}^{2} / 2}{\left[\left(1+\Gamma_{L}\right)^{4}-6\left(1+\Gamma_{L}\right) \Gamma_{0}\right]^{3 / 2}} .
\end{gathered}
$$

Here $\Gamma_{3}^{*}$ and $U_{1}^{*}$ are stable fixed points while the rest are unstable (Fig. (7).

The discriminants of Eq. (63) and Eq. (64) show that the critical applied stress in this case, $\sigma_{0}^{\text {crit } \prime}$ (or, $\Gamma_{0}^{\text {crit } \prime}$ ), is given by:

$$
\Gamma_{0}^{\mathrm{crit} \prime}=\frac{\sigma_{0}^{\mathrm{crit} \prime}}{\sigma_{R}-\sigma_{L}}=\frac{4}{27}\left(1+\Gamma_{L}\right)^{3}
$$

or,

$$
\sigma_{0}^{\text {crit } \prime}=\frac{4 \sigma_{R}^{3}}{27\left(\sigma_{R}-\sigma_{L}\right)^{2}} .
$$

In order to satisfy the condition $\sigma_{0}^{\text {crit } \prime} \geq \sigma_{L}$, it requires from Eq. (76) that

$$
\sigma_{R} \geq 3 \sigma_{L}
$$

which imposes an upper bound:

$$
\sigma_{0}^{\text {crit } \prime} \leq \frac{\sigma_{R}}{3}
$$

Like before, for $\Gamma_{0} \leq \Gamma_{0}^{\text {crit ' }}$ the stable fixed points are real-valued, which indicates that only partial failure of the fiber bundle takes place before a state of mechanical equilibrium is reached; for $\Gamma_{0}>\Gamma_{0}^{\text {crit }}$ ' the fixed points are not real and a phase of total failure exists. The order parameter $\mathcal{O}$ of the transition is given by the definition in Eq. (51). For $\Gamma_{0}=\Gamma_{0}^{\text {crit ' }}$ we get the following properties from Eq. (69), Eq. (170), Eq. (71) and Eq. (74):

$$
U_{1-\mathrm{crit}}^{*}=U_{2-\mathrm{crit}}^{*}=\frac{4}{9}\left(1+\Gamma_{L}\right)^{2}
$$


(a)

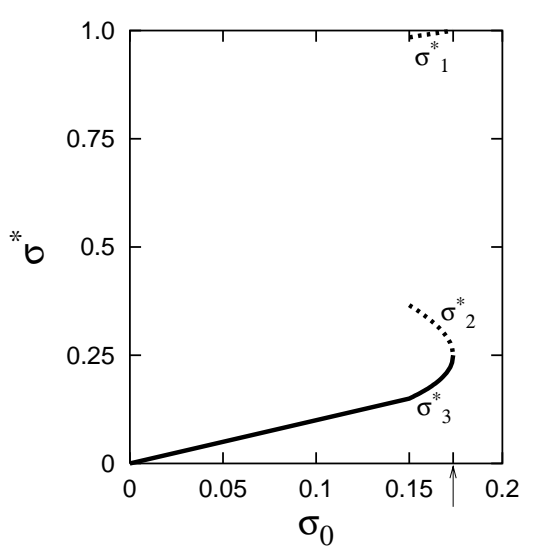

(b)

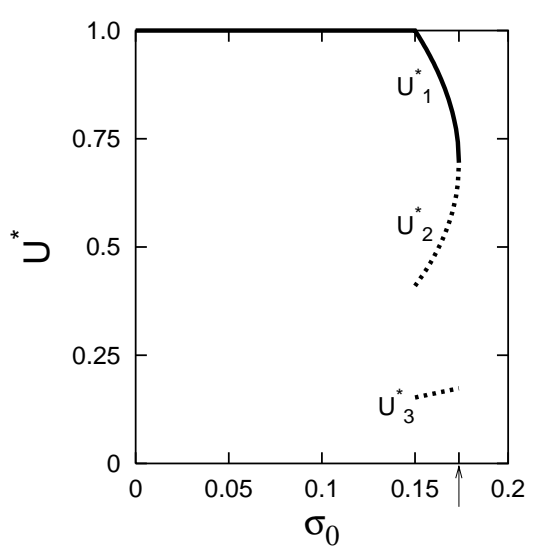

FIG. 7: The fixed points of (a) the redistributed stress, and (b) the surviving fraction of fibers for the probability distribution of fiber strengths shown in Fig. [6] The curve for the stable fixed points is shown by a bold solid line and those for the unstable fixed points are shown by bold broken lines. In this example too we have $\sigma_{L}=0.15$ and $\sigma_{R}=0.75$; here $\sigma_{0}^{\text {crit }}=0.173611$, marked by an arrowhead. The critical point is located lower than that in Fig. 5 due to abundance of fibers of lower strengths compared to the previous case.

$$
\cos \Theta_{\text {crit }}^{\prime}=-1 \quad \text { or, } \quad \Theta_{\text {crit }}^{\prime}=\pi
$$

and

$$
\cos \Phi_{\text {crit }}^{\prime}=1 \quad \text { or, } \quad \Phi_{\text {crit }}^{\prime}=0 .
$$

Comparing Eq. (81) and Eq. (80) with Eq. (50) we see that the critical values of $\Theta$ and $\Theta^{\prime}$ are the same whereas those of $\Phi$ and $\Phi^{\prime}$ differ by $\pi$ radians.

Near the critical point, but below it, we get from Eq. (70) and Eq. (71):

$$
U_{1}^{*} \simeq U_{1-\text { crit }}^{*}+\frac{4}{3}\left(1+\Gamma_{L}\right)^{1 / 2}\left(\Gamma_{0}^{\mathrm{crit} \prime}-\Gamma_{0}\right)^{1 / 2}
$$

and

$$
U_{2}^{*} \simeq U_{2-\text { crit }}^{*}-\frac{4}{3}\left(1+\Gamma_{L}\right)^{1 / 2}\left(\Gamma_{0}^{\mathrm{crit} \prime}-\Gamma_{0}\right)^{1 / 2}
$$

Therefore, by the definition of the order parameter in Eq. (51) and that of the susceptibility in (57), we get in this case $\mathcal{O} \propto\left(\Gamma_{0}^{\text {crit } \prime}-\Gamma_{0}\right)^{1 / 2}$ and $\chi \propto\left(\Gamma_{0}^{\text {crit } \prime}-\Gamma_{0}\right)^{-1 / 2}, \Gamma_{0} \rightarrow \Gamma_{0}^{\text {crit }}-$. These power laws have the same exponents as the corresponding ones in the previous cases and differ from those only in the critical point and the critical amplitude.

At the critical point the asymptotic relaxation of the surviving fraction of fibers to its stable fixed point [obtained as an asymptotic solution to (62)] is again found to be a power law decay similar to Eq. (28) and Eq. (58):

$$
\begin{aligned}
U_{t}-U_{1-\text { crit }}^{*} & \sim \frac{4}{3} \frac{U_{1-\text { crit }}^{*}}{t}, \quad t \rightarrow \infty \\
& \sim \frac{16}{27}\left(1+\Gamma_{L}\right)^{2} \frac{1}{t} .
\end{aligned}
$$


The critical behaviour of the models reported in this and the previous two sections show that the power laws found here are independent of the form of the probability distribution $P$. The three probability distributions studied have a common feature: the function $\sigma^{*}\left[1-P\left(\sigma^{*}\right)\right]$ has a maximum in the interval $\left(\sigma_{L}, \sigma_{R}\right)$ which corresponds to the critical value of the initial applied stress. All probability distributions having this property are therefore expected to lead to the same universality class as the three studied here. If the probability distribution does not have this property we may not observe a phase transition at all. For example, consider a fiber bundle model with $P\left(\sigma_{\text {thresh }}\right)=1-1 / \sigma_{\text {thresh }}, \sigma_{\text {thresh }} \geq 1$. Here $\sigma^{*}\left[1-P\left(\sigma^{*}\right)\right]=1$ and the evolution of the fiber bundle is given by the recursion relation $U_{t+1}=U_{t} / \sigma_{0}$ which implies that there is no dynamics at all for $\sigma_{0}=1$ and an exponential decay to complete failure, $U_{t}=\left(\sigma_{0}\right)^{-t}$, for $\sigma_{0}>1$. There are no critical phenomena and therefore no phase transition. However this general conclusion may not be true for finite-sized bundles [24].

\section{DISCUSSION}

In this paper we have studied the critical properties of failure in a class of fiber bundle models under an applied stress. The models are simple dynamical systems that show an irreversible phase transition. We have determined the static and dynamic critical properties associated with the phase transition. Since the models have been defined without any fluctuations in the local density of fiber strengths or in the load sharing, these are equivalent to a meanfield theory. We have defined a new order parameter in Eq. (19) which shows that the transition is of second-order. It is supported by facts which are characteristic of second-order transitions: the susceptibility diverges at the critical point and the decay of surviving fraction of fibers with time at the critical point follows a power-law. Besides, the ralaxation time of the bundle diverges from both sides of the critical point: for an initial stress infinitesimally below the critical point the surviving fraction of fibers will take an infinite time to reach its stable fixed point, whereas infinitesimally above the critical point it will take an infinite time to get past the fixed point. By obtaining the same critical exponents for three different probability distrbutions of fiber strengths, we are inclined to conclude that these exponents are universal. The universality of the critical exponent for susceptibility was also reported in Ref. [19, 20].

The two models studied in sections 4 and 5 seem to be related by the fact that their density functions, Eq. (29) and Eq. (59), can be transformed from one to the other by a reflection on the line $\sigma_{\text {thresh }}=\left(\sigma_{L}+\sigma_{R}\right) / 2$ (compare Fig. [4(a) and Fig. 6(a)). But the fixed point equations and their solutions do not have this symmetry. This is because the density function $\rho\left(\sigma_{\text {thresh }}\right)$ does not appear directly in the recursion relations for the dynamics. It is the distribution function $P\left(\sigma_{\text {thresh }}\right)$ which appears in the recursion relations. Eq. (30) and Eq. (60) show that the distribution functions of these two models are not mutually symmetric about any value of the threshold stress $\sigma_{\text {thresh }}$ (compare Fig. 4(b) and Fig. 6 b)). However a certain relation exists between the critical values of the applied stress for a special case of these two models: if $\sigma_{L}=0$, we get from Eq. (46) and Eq. (75) that $\sigma_{0}^{\text {crit }} / \sigma_{R}=\sqrt{4 / 27}$ and $\sigma_{0}^{\text {crit } \prime} / \sigma_{R}=4 / 27$ respectively; therefore we have $\sigma_{0}^{\text {crit } ~} / \sigma_{R}=\left(\sigma_{0}^{\text {crit }} / \sigma_{R}\right)^{2}$.

Finally we compare the fiber bundle model studied in this paper with the mean-field Ising model. Though the order parameter exponent (equal to $\frac{1}{2}$ ) of this model is identical to that of the mean-field Ising model the two models are not in the same universality class. The susceptibility in these models diverge with critical exponents $\frac{1}{2}$ and 1 respectively on approaching the critical point. The dynamical critical exponents are not the same either: in this fiber bundle model the surviving fraction of fibers under the critical applied stress decays toward its stable fixed point as $t^{-1}$, whereas the magnetization of the mean-field Ising model at the critical temperature decays to zero as $t^{-1 / 2}$.

[1] H. E. Daniels, Proc. R. Soc. London, Ser. A 183, 405 (1945).

[2] B. D. Coleman, J. Appl. Phys. 29, 968 (1958).

[3] Statistical Models for the Fracture of Disordered Media, edited by H. J. Herrmann and S. Roux (North-Holland, Amsterdam, 1990).

[4] B. K. Chakrabarti and L. G. Benguigui, Statistical Physics of Fracture and Breakdown in Disordered Systems (Clarendon Press, Oxford, 1997).

[5] D. Sornette, J. Phys. A: Math. Gen. 22, L243 (1989).

[6] P. C. Hemmer and A. Hansen, J. Appl. Mech. 59, 909 (1992).

[7] D. G. Harlow and S. L. Phoenix, Int. J. Frac. 17, 601 (1981).

[8] W. I. Newman, D. L. Turcotte, and A. M. Gabrielov, Phys. Rev. E 52, 4827 (1995).

[9] S-d. Zhang and E-j. Ding, Phys. Rev. B 53, 646 (1996).

[10] S-d. Zhang, Phys. Rev. E 59, 1589 (1999).

[11] W. I. Newman and S. L. Phoenix, Phys. Rev. E 63, 021507 (2001).

[12] L. Moral, Y. Moreno, J. B. Gomez, and A. F. Pacheco, Phys. Rev. E 63, 066106 (2001). 
[13] A. Hansen and P. C. Hemmer, Phys. Lett. A 184, 394 (1994).

[14] M. Kloster, A. Hansen, and P. C. Hemmer, Phys. Rev. E 56, 2615 (1997).

[15] R. C. Hidalgo, F. Kun, and H. J. Herrmann, Phys. Rev. E 64066122 (2001).

[16] S. Zapperi, P. Ray, H. E. Stanley, and A. Vespignani, Phys. Rev. Lett. 78, 1408 (1997).

[17] S. L. Phoenix, Adv. Appl. Prob. 11, 153 (1979).

[18] J. V. Andersen, D. Sornette, and K-t. Leung, Phys. Rev. Lett. 78, 2140 (1997).

[19] R. da Silveira, Phys. Rev. Lett. 80, 3157 (1998).

[20] R. da Silveira, Am. J. Phys. 67, 1177 (1999).

[21] Y. Moreno, J. B. Gomez, and A. F. Pacheco, Phys. Rev. Lett. 85, 2865 (2000).

[22] For a dynamical variable $X$ evolving by a recurrence of the form $X_{t+1}=F\left(X_{t}\right)$, the fixed points $X^{*}$ are the solutions of the equation $X^{*}=F\left(X^{*}\right)$. A fixed point $X^{*}$ is stable (at least a local attractor) if $|\mathrm{d} F / \mathrm{d} X|_{X=X^{*}} \leq 1$, otherwise it is unstable (a repeller); see R. M. May, Nature 261, 459 (1976).

[23] S. Pradhan, P. Bhattacharyya, and B. K. Chakrabarti, Phys. Rev. E 66, 016116 (2002).

[24] L. N. McCartney and R. L. Smith, J. Appl. Mech. 105, 601 (1983). 